\title{
Clinical outcome of SARS-CoV-2 infection in immunosuppressed children in Spain
}

\author{
Antonio Pérez-Martinez ${ }^{1}$ - Pilar Guerra-García ${ }^{1} \cdot$ Marta Melgosa $^{2}$ - Esteban Frauca ${ }^{3}$ - Carlota Fernandez-Camblor ${ }^{2}$. \\ Agustin Remesal $^{4} \cdot$ Cristina Calvo $^{5,6}$ (D)
}

Received: 5 May 2020 /Revised: 6 July 2020 / Accepted: 25 August 2020 / Published online: 29 August 2020

(C) Springer-Verlag GmbH Germany, part of Springer Nature 2020

\begin{abstract}
Limited information is available regarding SARS-CoV-2 infections in children with underlying diseases. A retrospective study of children less than 15 years old with primary or secondary immunosuppression infected with SARSCoV-2 during March 2020 was performed. In this series, 8 immunocompromised patients with COVID-19 disease are reported, accounting for $15 \%$ of the positive cases detected in children in a reference hospital. The severity of the symptoms was mild-moderate in the majority with a predominance of febrile syndrome, with mild radiological involvement and in some cases with mild respiratory distress that required oxygen therapy. The rational and prudent management of these patients is discussed, evaluating possible treatments and options for hospitalization or outpatient follow-up.

Conclusion: In our experience, monitoring of children with immunosuppression and COVID-19 disease can be performed as outpatients if close monitoring is possible. Hospitalization should be assessed when high fever, radiological involvement, and/or respiratory distress are present.
\end{abstract}

What is Known:

- SARS-CoV-2 infection is usually mild in children.

What is New:

- Outcome of immunosuppressed children with COVID-19 is generally good, with a mild-moderate course.

Keywords COVID-19 $\cdot$ Transplant $\cdot$ Cancer $\cdot$ Immunosuppression

Communicated by Nicole Ritz

Cristina Calvo

ccalvorey@gmail.com

Antonio Pérez-Martinez

antonioperezmartinez@yahoo.es

Pilar Guerra-García

pilar.guerra@salud.madrid.org

Marta Melgosa

marta.melgosa@salud.madrid.org

Esteban Frauca

esteban.frauca@salud.madrid.org

Carlota Fernandez-Camblor

carlotam.fernandez@salud.madrid.org

Agustin Remesal

agusremesal@hotmail.com
1 Pediatric Hemato-Oncology Department, Hospital Universitario La Paz, Madrid, Spain

2 Pediatric Nephrology Department, Hospital Universitario La Paz, Madrid, Spain

3 Pediatric Hepatology Department, Hospital Universitario La Paz, Madrid, Spain

4 Pediatric Rheumatology Department, Hospital Universitario La Paz, Madrid, Spain

5 Pediatric and Infectious Diseases Department, Hospital Universitario La Paz, Paseo de la Castellana, 26128046 Madrid, Spain

6 Fundación IdiPaz, Translational Research Network in Pediatric Infectious Diseases (RITIP), Madrid, Spain 


$\begin{array}{ll}\text { Abbreviations } \\ \text { ACE2 } & \text { Angiotensin-converting enzyme 2 } \\ \text { ESPGHAN } & \begin{array}{l}\text { European Society for Gastroenterology } \\ \text { sHLH }\end{array} \\ & \begin{array}{l}\text { Hemophagocytic } \\ \text { lymphohistiocytosis-like syndrome }\end{array} \\ \text { SIOP } & \begin{array}{l}\text { International Society of Pediatric } \\ \text { Oncology }\end{array} \\ \text { IQR } & \text { interquartile range } \\ \text { PCR } & \text { Polymerase chain reaction }\end{array}$

\section{Introduction}

SARS-CoV-2 infection in children younger than 10 years of age has been described in around $1 \%$ of identified cases in China [1]. Although data on children is still limited, most series agree that cases are mostly mild, even in infants $[2,3]$. Very little information is available about pediatric patients with underlying diseases, and data in children with immunosuppression is almost non-existent. COVID-19 serious disease has been described in a child with post-chemotherapy agranulocytosis due to acute leukemia [4]. Preliminary data from Bergamo [5] indicates that in Italy, practically no cases of infection have been detected in immunosuppressed patients ( 3 mild cases), which has led them to consider that this group of patients is not a high-risk group for COVID-19 disease.

Spain is currently one of the most affected countries with more than 100,000 reported cases. The first case in Spain was declared on January 31st, 2020, and the first case in Madrid was declared on February 27th, 2020. La Paz University Hospital in Madrid, Spain, is a national reference tertiary center for transplanted, oncological, and immunosuppressed children. Over 1,300 pediatric solid organ (SOT) and 630 hematopoietic stem cell transplants (HSCT) have been performed in our hospital, and more than 3,200 cancer patients have been treated over more than five decades. Per year, we diagnose 100-120 new pediatric cancer patients, and perform 35 HSCT and 65 SOT. Our aim was to describe the children with primary or secondary immunosuppression, and SARS-CoV-2 infection attended in our hospital during the month of March.

\section{Patients and methods}

A retrospective study (1st to 31st of March, 2020) of children less than 15 years old with primary or secondary immunosuppression infected with SARS-CoV-2 and treated at the University Hospital La Paz, Madrid, Spain, was performed. For inclusion in the study, the SARS-CoV-2 polymerase chain reaction (PCR) had to be positive. PCR was performed via nasal swabs, and if the test was initially negative but clinical suspicion was high, it was repeated in the next $24 \mathrm{~h}$. Inpatients and outpatients were included.
Symptoms, blood analysis results and radiological data, previous underlying disease, immunosuppressive treatment, treatment received for COVID-19 disease, and evolution were collected.

\section{Results}

During the month of March, a total of 315 children were tested in our hospital for SARS-CoV-2 and 51 were positive (16\%). Twenty-eight of the positive cases $(55 \%)$ were admitted. A total of 8 positive cases $(15 \%)$ were immunosuppressed patients, and 3 were followed as outpatients. The underlying pathology was hemato-oncological in most cases (three patients had undergone a hematopoietic stem cell transplant), two patients had undergone a liver or a kidney transplant, and the last case was a girl on chronic hemodialysis with a vasculitis receiving immunosuppressive treatment. The median age was 12.6 years (interquartile range: IQR 9.5-12.6). All the patients had been on immunosuppressive treatment with a median duration of 24 months (IQR 12-93). All had respiratory symptoms, 5 of them had fever, and the majority had slight radiological alterations (only two had focal infiltrates). In only one case the chest X-ray worsened during hospitalization. One patient needed oxygen therapy and one case had diarrhea. None of them had anosmia or ageusia. One patient developed a hemophagocytic lymphohistiocytosis-like syndrome (sHLH), with persistent fever, cytopenias, low fibrinogen and elevated triglycerides, and elevated ferritin, and required specific treatment with dexamethasone, as per the HLH-2004 protocol. Antiviral treatment was initiated according to our National guidelines, developed by the National Infectious Disease Society (SEIP), in coordination with the National Pediatric Intensive Care Society (SECIP) and the National Pediatric Association (AEP) available at https:// www.aeped.es/noticias/documento-manejo-clinico-pacientepediatrico-y-pacientes-riesgo-con-infeccion-por-sars-cov2. Hydroxychloroquine was initiated upon diagnosis (positive PCR for SARS-CoV-2) when there were signs/ symptoms of moderate-severe disease (respiratory signs, pneumonia on chest X ray, blood parameters of severity such as lymphopenia or elevated CRP, D-dimer or Il-6). Remdesivir was requested, under compassionate use, in the most severe cases. Following theses guidelines, in 5/8 cases, some antiviral treatment was prescribed, with hydroxychloroquine being the most frequent. Two patients received remdesivir (the two oncological patients who required admission: patient 1 who had oxygen requirement and patient 4 who had persistent symptoms with severe lymphopenia and elevated inflammatory parameters) and only one patient received tocilizumab due to persistent fever and elevated IL-6. There were no significant toxicities following the treatments received. Immunosuppressive therapy was decreased or withdrawn in 6 patients (the other two 
had been off immunosuppression for 1 and 2 months, respectively). All but one case developed progressive lymphopenia. D-dimer was moderately elevated. With a median follow up of 106 days (IQR 103-113) from COVID-19 diagnosis, no child required admission to the pediatric intensive care unit or died. At present, all the patients are being followed up as outpatients and have had no further complications related to COVID-19. The clinical characteristics of the patients, as well as details on the treatment received, are outlined in Table 1.

\section{Discussion}

In this small series, 8 immunocompromised patients with COVID-19 disease are reported, accounting for $15 \%$ of the positive cases detected in children in a reference hospital. The severity of the symptoms was mild-moderate in the majority with a predominance of febrile syndrome, with mild radiological involvement and in some cases with mild respiratory distress that required oxygen therapy. Most patients received antiviral treatment, and immunosuppressive treatment was decreased or even discontinued for a short period of time in most of them.

It has been described by most of the authors that children have a milder disease than adults $[2,3]$ and immunocompromised patients do not seem to evolve differently [5]. Data in children is practically non-existent. Recently, a study carried out by the European Society for Gastroenterology (ESPGHAN), described children with inflammatory bowel disease who presented COVID-19 disease with a mild outpatient course [6]. In addition, they warned of the risk of developing acute exacerbations of the disease if immunosuppressant treatments were delayed. Data from cancer pediatric patients is scarce. A recent report from a childhood cancer center in Lombardy, Italy, reported five positive cases in childhood cancer patients and all of them had a mild course [7]. In fact, the International Society of Pediatric Oncology (SIOP) highlighted that there is no reason to discontinue daily activities in pediatric hematology/ oncology units; however, general principles for prevention were recommended [8].

SARS-CoV-2 infection is proposed to evolve in three phases, causing mortality in the third phase, after 2 or more weeks from symptom onset [9]. During the early phase, SARS-CoV-2 multiplies and establishes residence in the host, primarily focusing on the respiratory system. SARS-CoV-2 binds to its target using the angiotensinconverting enzyme 2 (ACE2), receptor on human cells [10] mainly in human lung, small intestine epithelium, as well as the vascular endothelium. This phase presents with mild respiratory symptoms. During the second phase, lung involvement is established, with viral pneumonia and the development of hypoxia. Lymphopenia appears or increases and a moderate increase in inflammatory markers can be observed. A minority of patients will reach the third phase of systemic hyperinflammation. There is a decrease in helper, suppressor, and regulatory $\mathrm{T}$ cell counts, with increase of inflammatory cytokines and biomarkers such as interleukins IL-2, IL-6, IL-7, granulocyte-colonystimulating factor, macrophage inflammatory protein $1-\alpha$, tumor necrosis factor- $\alpha, C$-reactive protein, ferritin, and Ddimer [11]. A hemophagocytic lymphohistiocytosis-like syndrome (sHLH) may occur in patients in this advanced stage of disease.

The possibility that immunosuppressed patients may not be able to progress to advanced stages of the disease could explain why most behave with mild symptoms, as previously reported [5]. Although our patients presented lymphopenia, most of them did not have significantly increased D-dimer or Il-6 when it was performed. Despite two patients having elevated D-dimer, anticoagulation treatment was not given, as it is not recommended by our Spanish Infectious Disease Society based on the available evidence. One of our patients developed sHLH that was treated with dexamethasone as per the HLH-2004 trial and entered remission. The patient finished the course of steroids, and had no signs of recurrence. Therefore, although most children have a mild-moderate course, it is important to be aware of possible complications that require prompt intervention.

A problem that must be assessed is the balance between risk and benefit of the suspension of the immunosuppressive treatment. We believe that risk has to be individually assessed, as well as the necessity to start specific antiviral treatments.

Based on our experience, monitoring of children with immunosuppression and COVID-19 disease can be performed as outpatients, if close monitoring is possible with radiological and blood test controls if necessary, and carefully selecting the patients depending on their individual risk. Hospitalization should be assessed when high fever, radiological involvement, and/or respiratory distress is present. Also, it should be considered if there are signs of disease severity (lymphopenia, elevated D-dimer, Creactive protein or Interleukin-6). Depending on the evolution, an sHLH must be ruled out. A judicious and conservative treatment seems safe and recommended since to date no antiviral treatment has shown efficacy. It is particularly relevant to be very careful when recommending any specific antiviral treatment in this group of patients given the numerous drug interactions that may appear with their immunosuppressive treatment, such as calcineurin inhibitors, among others. The available tables of pharmacological interactions should always be consulted.

Other groups should communicate their experience with these patients to offer appropriate treatment and follow-up. 


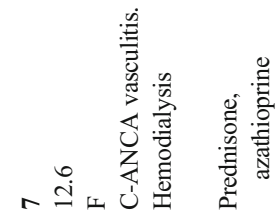

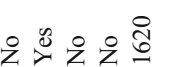

8 กี

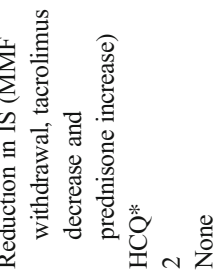

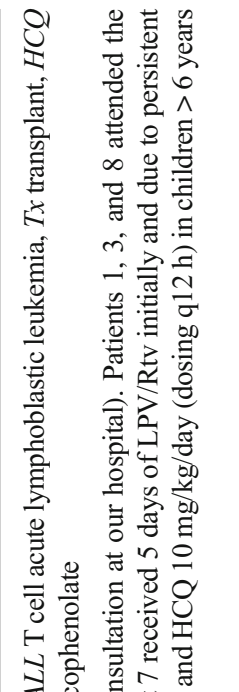

암

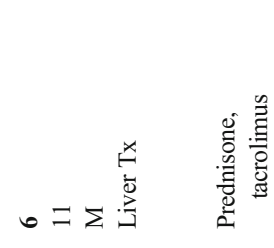

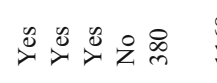

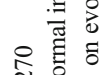

童

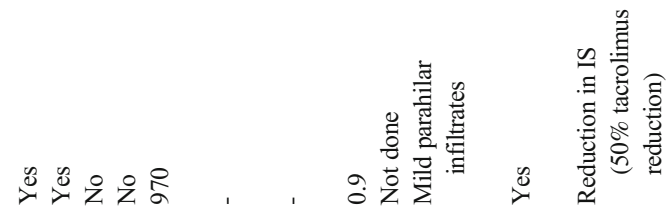

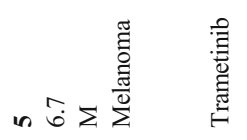

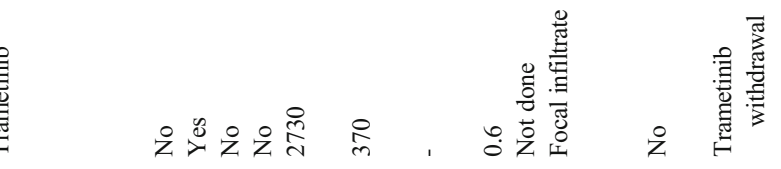

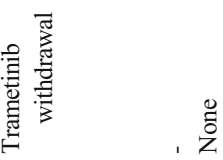

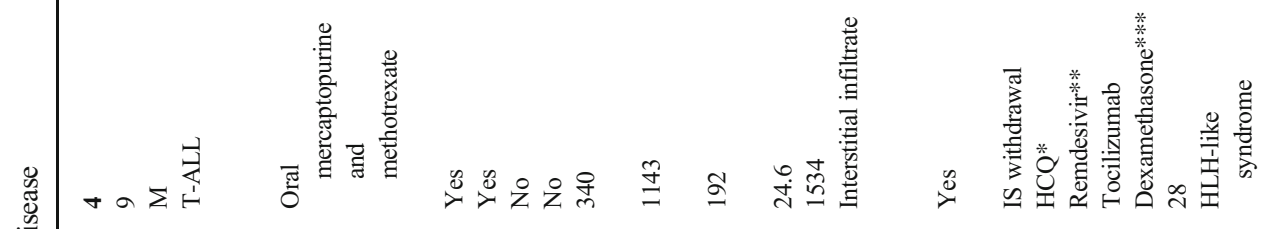

字

官

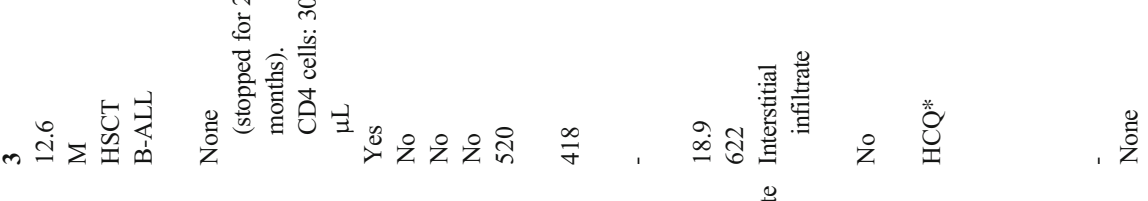

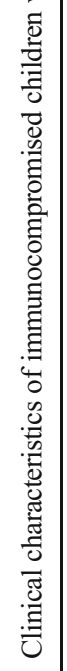

ง

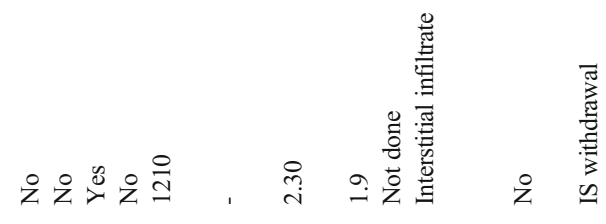

そั

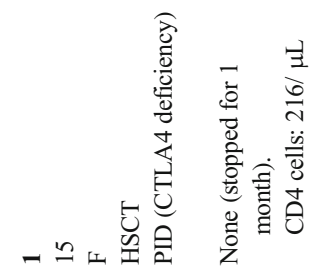

, :

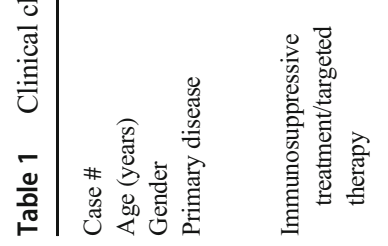

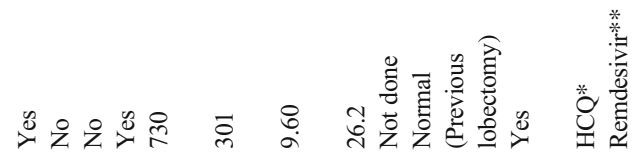

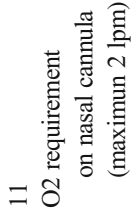

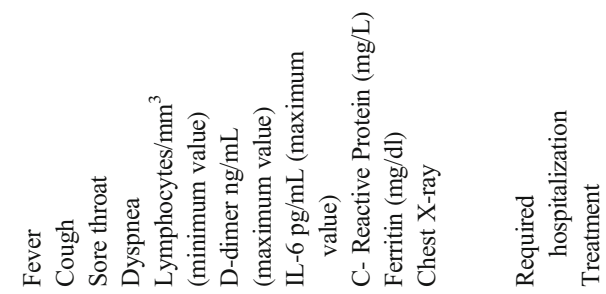

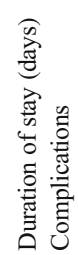

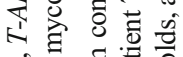

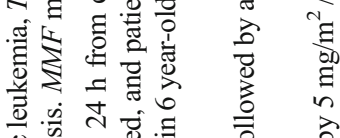

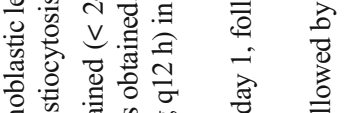

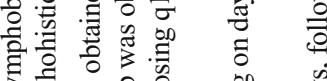

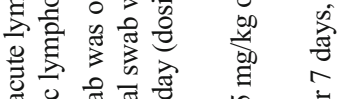

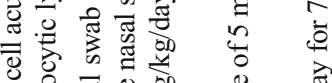

๑ :

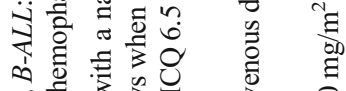

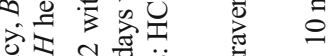

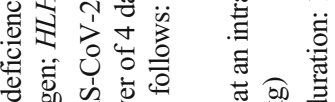

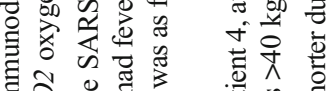

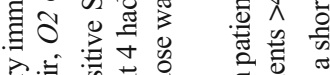

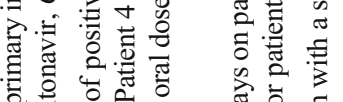

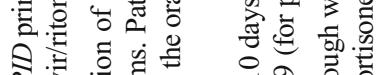

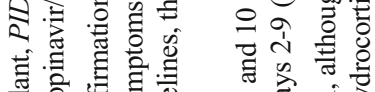

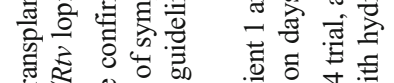

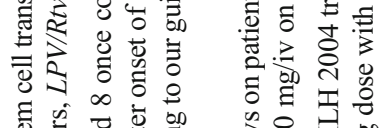

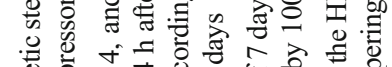

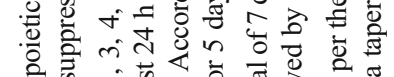

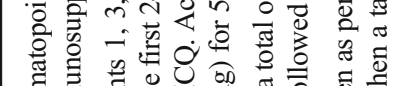

들

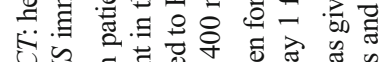

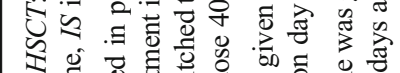

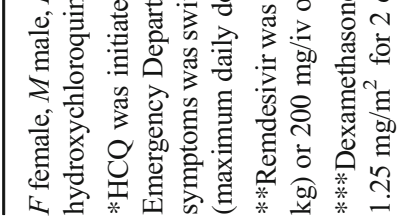


Author Contribution Dr. P-M and Dr. M conceptualized and designed the study, drafted the initial manuscript, and reviewed and revised the manuscript. Dr. F, Dr. F-C, Dr. R, Dr. G collected data, collaborated in drafted the initial manuscript and reviewed and revised the manuscript. Dr. C designed the study, coordinated and supervised data collection, collaborated in drafted the initial manuscript and critically reviewed the manuscript. All authors approved the final manuscript as submitted and agree to be accountable for all aspects of the work.

\section{Compliance with ethical standards}

Conflict of interest The authors declare that they have no conflicts of interest.

\section{References}

1. Wu Z, McGoogan JM (2020) Characteristics of and important lessons from the coronavirus disease 2019 (COVID-19) outbreak in China: summary of a report of 72314 cases from the Chinese Center for Disease Control and Prevention. JAMA. https://doi. org/10.1001/jama.2020.2648

2. MinWei MD, Yuan J, Liu Y, Fu T, Yu X, Zhang ZJ (2020) Novel coronavirus infection in hospitalized infants under 1 year of age in China. JAMA. Published online February 14. https://doi.org/10. 1001/jama.2020.2131.20

3. Dong Y, Mo X, Hu Y, Qi X, Jiang F, Jiang Z, Tong S (2020) Epidemiological characteristics of 2143 pediatric patients with 2019 coronavirus disease in China. Pediatrics. 145:e20200702. https://doi.org/10.1542/peds.2020-0702

4. Chen Z, Xiong H, Li JX et al (2020) COVID-19 with postchemotherapy agranulocytosis in childhood acute leukemia: a case report. 41(0):E004. https://doi.org/10.3760/cma.j.issn.0253-2727. 2020.0004

5. D'Antiga L (2020) Coronaviruses and immunosuppressed patients. The facts during the third epidemic. Liver Transplant. https://doi. org/10.1002/LT.25756

6. Turner D, Huang Y, Martín-de-Carpi J et al (2020) COVID-19 and paediatric inflammatory bowel diseases: global experience and provisional guidance (March 2020) from the Paediatric IBD Porto group of ESPGHAN. J Pediatr Gastroenterol Nutr 2020. https:// doi.org/10.1097/MPG.0000000000002729

7. Balduzzi A, Brivio E, Rovelli A, Rizzari C, Gasperini S, Melzi ML, Conter V, Biondi A (2020) Lessons after the early management of the COVID-19 outbreak in a paediatric transplant and haematooncology centre embedded within a COVID-19 dedicated hospital in Lombardia, Italy. Bone Marrow Transplant (in press)

8. Bouffet E, Challinor J, Sullivan M, Biondi A, Rodriguez-Galindo C, Pritchard-Jones K (2020) Early advice on managing children with cancer during the COVID-19 pandemic and a call for sharing experiences. Pediatr Blood Cancer. 2020:e28327. https://doi.org/ $10.1002 /$ pbc. 28327

9. Siddiqi HK, Mehra MR. COVID-19 Illness in native and immunosuppressed states: a clinical-therapeutic staging proposal. J Heart Lung Trasplant In press.

10. Wan Y, Shang J, Graham R, Baric RS, Li F (2020) Receptor recognition by novel coronavirus from Wuhan: an analysis based on decade-long structural studies of SARS. J Virol 94. https://doi.org/ 10.1128/jvi.00127-20

11. Mehta P, McAuley DF, Brown M, Sanchez E, Tattersall RS, Manson JJ COVID-19: consider cytokine storm syndromes and immunosuppression. Lancet. https://doi.org/10.1016/S01406736(20)30628-0

Publisher's note Springer Nature remains neutral with regard to jurisdictional claims in published maps and institutional affiliations. 